\title{
Espalhamento Bhabha: Um Estudo Detalhado
}

\author{
Bhabha Scattering: A Detailed Study
}

\author{
Fábio Köpp Nóbrega*1@, Luiz Fernando Mackedanz ${ }^{2}$ \\ ${ }^{1}$ Universidade Federal do Rio Grande do Sul, Instituto de Física, Porto Alegre, RS, Brasil \\ ${ }^{2}$ Universidade Federal do Rio Grande, Instituto de Matemática, Estatística e Física, Programa de Pós-Graduação em \\ Educação em Ciências, Rio Grande, RS, Brasil
}

Recebido em 18 de Dezembro, 2017. Revisado em 26 de Fevereiro, 2018. Aceito em 14 de Março, 2018.

\begin{abstract}
Neste artigo, vamos estudar alguns conceitos fundamentais em física de partículas através do estudo detalhado de um processo específico da Eletrodinâmica Quântica (QED): o espalhamento Bhabha em ordem dominante (Leading Order - LO). Este ocorre na interação entre um elétron e sua antipartícula, o pósitron, sendo um dos processos básicos da QED. Nossa escolha em trabalhar este processo deve-se a riqueza de detalhes proporcionada pelas duas possibilidades (canais) de interação, que servem para ilustrar o cálculo da interferência entre as possibilidades. Além disso, esse processo é utilizado para determinar a luminosidade de um determinado colisor, o que garante maior precisão nas medidas de outras grandezas relevantes para a análise das interações entre partículas. Finalmente, comparamos a predição da QED com os resultados do experimento DESY-PETRA-TASSO. Palavras-chave: física de partículas, processos elementares, eletrodinâmica quântica.
\end{abstract}

In this article, we will look at some fundamental concepts in Particle Physics through the detailed study of a specific process of Quantum Electrodynamics (QED): the Bhabha scattering. It occurs in the interaction between an electron and its antiparticle, the positron, one of the basic QED processes. Our choice for work this process is due to the wealth of details provided by the two possibilities (channels) of interaction which serve to illustrate the calculation of interference between possibilities. Furthermore, this process is used to determine the luminosity of a particular collider, which ensures greater accuracy in measurements of other relevant quantities for the analysis of interactions between particles. Finally, comparing the QED prediction with the experimental results of the DESY-PETRA-TASSO experiment.

Keywords: particle physics, elementary processes, quantum electrodynamics.

\section{Introdução}

A divulgação científica de física de partículas, apesar das notícias apresentadas pela mídia nacional e internacional da descoberta do Bóson de Higgs pelo CERN, ainda não possui muitos livros em português. O que dificulta um pouco o interesse de alunos de graduação por tal tema. No entanto, há dois livros de divulgação científica, em português, de pesquisadores nesta área: "O Discreto Charme das Partículas Elementares"da Professora Maria Cristina Batoni Abdalla, destinado a pessoas leigas em física. E "O Cerne da Matéria"do Professor Rogério Rosenfeld, o qual relata toda a trajetória dos físicos e experimentos que culminaram na descoberta do Bóson de Higgs noticiada no dia 4 de Julho de 2012 pelo CERN. O livro deste professor é recomendado tanto para físicos de outras áreas quanto propriamente para pesquisadores desta área. Assim, a principal motivação deste artigo é fornecer um material em português bem como os códigos e scripts necessários para reproduzir os valores e gráficos aqui apresentados. Por isso, direcionamos este texto para estudantes de graduação, nos primeiros anos,

*Endereço de correspondência: fabio.kopp@ufrgs.br que tenham interesse em aprofundar conhecimentos na física de partículas, além de buscar dar uma base para a Iniciação Científica ao longo da graduação.

O estudo de processos básicos do Modelo Padrão (Standard Model) ocorre, normalmente, nos últimos anos do bacharelado em física ou em disciplinas específicas de pós-graduação. Neste sentido, buscamos proporcionar ao aluno uma visão geral do processo Bhabha, bem como uma breve revisão sobre a teoria da Eletrodinâmica Quântica (QED: Quantum electrodynamics). O processo Bhabha, assim como outros processos da QED, possuem um "sinal"limpo quando comparado a processos que envolvam a colisão de núcleons (prótons e nêutrons). Em processos deste tipo, envolvendo energias maiores do que a massa destas partículas, a interação se dá a nível dos constituintes (quarks e glúons), produzindo uma miríade de novas partículas. A descrição da dinâmica destas interações é feita pela Cromodinâmica Quântica (QCD: Quantum Chromodynamics), associada a um novo número quântico: a carga de cor que cada quark pode portar. Esta pode ser comparada, de forma ingênua, com a carga elétrica, pois também possui dois tipos de carga: cor e anticor. Por outro lado, segundo a QCD, todos os estados 
ligados detectáveis devem ter um estado final branco - o chamado singleto de cor.

Um exemplo deste tipo de interação é realizado no Grande Colisor de Hádrons (LHC: Large Hadron Collider). As partículas produzidas nesse tipo de colisor possuem diferentes propriedades, tais como spin, massa, e carga elétrica. No momento da análise da colisão, diferenciar uma partícula $\mathrm{X}$ de todas as outras requer detectores específicos e simulações entre a estrutura do detector e a física subjacente. Ainda, devemos lembrar que processos envolvendo partículas e antipartículas também surgem nesse tipo de colisão, porém, desta vez, regidos pela teoria Eletrofraca: unificação da teoria eletromagnética (QED) com a teoria para as interações fracas, responsáveis pelo decaimento nuclear beta e também pelo decaimento do píon e do múon, entre outras. Desta forma, dizemos que processos analisados pela teoria eletrofraca resultam num sinal experimental com menos ruído. O Colisor Linear Internaciona ${ }^{1}$ (ILC: International Linear Collider) colidirá elétrons e pósitrons e utilizará o processo Bhabha para determinar a sua luminosidade [1]. Além disso, procurará determinar as propriedades do Bóson de Higgs, Dimensões extras e a partícula supersimétrica mais leve.

A utilidade mais próxima de nós do estudo desse tipo de processo pode ser visto em [2] o qual trata da antimatéria na medicina moderna. Por fim, devemos lembrar que há estudos igualmente importantes nesta área como estados ligados, decaimentos, oscilações de neutrinos e medidas de momentos dipolares magnéticos e elétricos.

Neste sentido, propomos trabalhar a transposição externa do conteúdo, aquela onde o pesquisador traz os conhecimentos da área para um nível acessível ao estudante interessado nesta área da Física, inserindo novas visões para temas já apresentados nos primeiros anos da graduação, ou novos conceitos apresentados pela primeira vez [3]. Nosso foco, neste artigo, é discutir fundamentos da Física de Partículas Elementares, através dos processos da Eletrodinâmica Quântica (QED) [4 9], haja vista que os processos básicos de interação entre elétrons, ou entre o elétron e sua antipartícula - o pósitron - são importantes em diversos ramos de aplicação da Física, como por exemplo a Física das Radiações [10], onde a aniquilação e produção de pares são utilizadas para o diagnóstico e tratamento de doenças. Este é o princípio da tomografia por emissão de pósitrons (PET). Em particular, vamos nos dedicar ao estudo de um processo que inclua estes dois aspectos: o processo Bhabha, que pode ser interpretado como uma aniquilação entre elétron e pósitron seguida da produção do par.

Por esse motivo, este texto básico se destina a estudantes de graduação interessados na fenomenologia dos processos da Física de Partículas, como uma leitura introdutória que apresenta as grandezas fundamentais envolvidas nestes. Para tanto, na seção 2, apresentaremos o princípio de Gauge aplicado a QED e sua aplicação ao processo Bhabha. No entanto, não vamos derivar o

1 https://www.linearcollider.org/ILC propagador do elétron e do fóton devido ao escopo deste trabalho, para maiores detalhes vide [11-14]. Já na seção 3 , fazemos uma revisão sobre algumas grandezas básicas utilizadas modernamente na Física de Partículas, bem como a atual forma de cálculo, através dos diagramas de Feynman. Na seção 4, apresentamos os cálculos do processo de forma detalhada, principalmente o termo de interferência, normalmente não incluído nos livros texto da área. Finalmente, elencamos algumas conclusões relevantes e aplicações do processo dentro das atuais colaborações nos aceleradores de partículas, como o ILC.

\section{Introdução à Eletrodinâmica Quântica}

A eletrodinâmica quântica (QED, do inglês Quantum Electrodynamics) é a teoria que unifica os fenômenos eletromagnéticos, como ondas de rádio e radiações, com a física quântica, levando a aplicações práticas na eletrônica, computação e descreve as interações entre quarks e léptons. Ainda, devemos salientar que a QED é quase toda a base da química que conhecemos. Além disso, é uma teoria Abeliana, ou seja, os campos de gauge comutam entre si $\left(\left[A_{\mu}, A_{\nu}\right]=0\right)$ pertencendo ao grupo $U(1)$, sendo estes os campos eletromagnéticos. Por Abeliano, dizemos que os mediadores de campo (os fótons), não possuem carga e, portanto, não podem interagir entre si. Nos diagramas de Feynman temos vértices, ponto de "contato", entre partículas/anti-particulas com o propagador do bóson que media a interação. Este vértice surge ao aplicarmos o princípio de Gaug ${ }^{2}$ nos campos fermiônico. Para termos uma ideia de como o obtemos, partimos do Lagrangeano para partícula livre de spin $1 / 2$ (Dirac) dado por

$$
\mathcal{L}_{\text {Dirac }}=\bar{\psi}\left(i \gamma^{\mu} \partial_{\mu}-m\right) \psi
$$

A transformação de gauge local (dependência de coordenadas tipos espaço em $\alpha(x))$ ocorre da seguinte forma:

$$
\psi^{\prime}(x) \rightarrow e^{i \alpha(x)} \psi(x) \text { e } \bar{\psi}^{\prime}(x) \rightarrow \bar{\psi}(x) e^{-i \alpha(x)}
$$

Vale lembrar que existem outros tipo de transformações de gauge, como a global. Em que $\alpha$, desta vez, não depende de coordenadas tipo espaço. Retornando a derivação, substituindo (2) em (1) e tomando a derivada parcial, obtemos:

$$
\partial_{\mu} \psi^{\prime} \rightarrow e^{i \alpha(x)} \partial_{\mu} \psi(x)+i e^{i \alpha(x)} \psi(x) \partial_{\mu} \alpha(x)
$$

O segundo termo de (3) quebra a invariância de $\mathcal{L}_{\text {Dirac }}$ Uma forma de contornar esse problema, é modificar a derivada parcial para derivada covariante de forma a satisfazer a invariância de gauge local

$$
\mathcal{D}_{\mu}^{\prime} \psi^{\prime} \rightarrow e^{i \alpha(x)} \mathcal{D}_{\mu} \psi
$$

\footnotetext{
$\overline{2 " A s ~ e q u a c ̧ o ̃ e s ~ d e ~ m o v i m e n t o ~ s a ̃ o ~ i n v a r i a n t e s ~ s o b ~ u m a ~ t r a n s f o r m a-~}$ ção de Gauge local".
} 
em que definimos

$$
\mathcal{D}_{\mu}^{\prime} \equiv \partial_{\mu}-i e \mathcal{A}_{\mu}^{\prime},
$$

onde $\mathcal{A}_{\mu}$ é um campo vetorial que transforma-se como:

$$
\mathcal{A}_{\mu}^{\prime} \rightarrow \mathcal{A}_{\mu}+\frac{1}{e} \partial_{\mu} \alpha(x)
$$

Refazendo o cálculo anterior, exceto por termos agora $\mathcal{D}_{\mu}$, obtemos

$$
\mathcal{D}_{\mu}^{\prime} \psi^{\prime}=\mathcal{D}_{\mu}\left(e^{i \alpha(x)} \psi\right)=\left(\partial_{\mu}-i e \mathcal{A}_{\mu}^{\prime}\right)\left(e^{i \alpha(x)} \psi\right) .
$$

Substituindo (6) em (7), vem

$$
\mathcal{D}_{\mu}^{\prime} \psi^{\prime}=\left[\partial_{\mu}-i e\left(\mathcal{A}_{\mu}+\frac{1}{e} \partial_{\mu} \alpha(x)\right)\right]\left(e^{i \alpha(x)} \psi\right) .
$$

Desta forma, encontramos

$$
\mathcal{D}_{\mu}^{\prime} \psi^{\prime}=e^{i \alpha(x)}\left(\partial_{\mu}-i e \mathcal{A}_{\mu}\right) \psi=e^{i \alpha(x)} \mathcal{D}_{\mu} \psi .
$$

Logo, a equação (9) satisfaz à condição (4) tornando-se, assim, invariante de gauge local.

Para podermos entender o processo de espalhamento Bhabha, bem como estender os conceitos para demais processos, precisamos inicialmente ter alguns conhecimentos e definições básicos sobre referenciais e grandezas próprias da Física de partículas. A próxima seção é dedicada a apresentar estes fundamentos, de forma breve. Eles podem ser encontrados, para quem desejar aprofundar seus estudos em 11,15 .

\section{Parâmetros em Física de Partículas}

Para estudarmos os processo básicos da Física de Partículas, em que no nosso caso é o espalhamentos, devemos conhecer a cinemática relativística, que descreve as grandezas dinâmicas e suas relações em altas energias. Nessa seção apresentaremos os principais parâmetros físicos necessários para compreendermos os processos simples, como o espalhamento Bhabha. Durante todo o trabalho, usaremos as unidades naturais: $\mathrm{c}=1$ e $\hbar=1$, da forma como usualmente é trabalhado na Física de Partículas. Isso permite que tratemos todas as grandezas cinemáticas em termos de unidades de energia, que na área é o elétron-Volt $(\mathrm{eV})$ e seus múltiplos.

Uma vez que os processos investigados envolvem partículas em altas velocidades, comparáveis à da luz $(v \approx c)$, devemos utilizar a Relatividade Restrita( $[6-18])$. Nesse contexto, precisamos definir inicialmente grandezas que sejam invariantes de referencial, ou seja, grandezas que tem um valor único, não importando o referencial no qual as computemos. Uma rápida inspeção em textos básicos sobre Relatividade Restrita nos mostra que estas grandezas podem ser relacionados como um 4-vetor, com 1 componente temporal adicionada às três espaciais. $\mathrm{O}$ exemplo clássico é o intervalo definido no espaço-tempo

$$
(\Delta s)^{2}=(\Delta t)^{2}-(\Delta x)^{2}-(\Delta y)^{2}-(\Delta z)^{2},
$$

que ilustra com bastante clareza as 4 componentes, diferenciadas pelo sinal negativo ante das coordenadas espaciais. Por conta deste sinal, dizemos que este espaçotempo possui uma métrica diagonal, ou seja, a matriz que representa tal espaço-tempo é representada por uma matriz apenas com elementos não nulos na diagonal principal. Esta é chamada de métrica de Minkowski com $\operatorname{diag}(1,-1,-1,-1)$.

Na análise dinâmica de um espalhamento, a grandeza generalizada será o 4-vetor momentum (cf. [19 22]), denotado por

$$
p^{\mu}=\left(p^{0}, p^{1}, p^{2}, p^{3}\right)=\left(E, p_{x}, p_{y}, p_{z}\right),
$$

a qual respeitar as transformações de Lorentz entre referenciais inerciais. Comumente os referenciais mais utilizados são o de laboratório (LAB) onde as partículas envolvidas têm seus momenta medidos em relação a algo externo à colisão e o referencial de centro de massa (CM), usado para facilitar as considerações das leis de conservação (momentum e energia). Deste último, falaremos um pouco mais pela sua praticidade para analisar os espalhamentos. Lembramos que, para estes processos, a conservação do 3 -vetor momentum é garantida.

No referencial de centro de massa, vamos considerar o processo esquematizado na Fig. 1 como uma colisão elástica, ou seja, $E_{\text {total }}=E_{1}+E_{2}$ também é conservada. Neste referencial, o momentum total inicial é nulo, ou seja, $\left(\mathbf{P}_{\text {total }}=\mathbf{P}_{1}+\mathbf{P}_{2}\right)=\mathbf{0}$. Isto significa que $\left.\mathbf{P}_{1}=-\mathbf{P}_{2}\right)$. Além disso, como estamos trabalhando com feixes de mesma energia, com partículas de massa similar (pósitrons e elétrons), consideramos $E_{1}=E_{2}=E_{c m} \mathrm{e}$ $P_{1}=P_{2}=P$.

Em termos dos 4-momenta, $P_{1}^{\mu}=\left(E_{1}, \mathbf{P}\right)$ e $P_{2}^{\mu}=$ $\left(E_{2},-\mathbf{P}\right)$. Para calcularmos um invariante associado ao 4vetor momentum, utilizamos a regra de soma de Einstein

$$
P^{2}=P^{\mu} P_{\mu}
$$

onde a mudança na posição do índice simboliza a troca da matriz que representa o 4 -vetor por sua transposta. $\mathrm{O}$ produto escalar entre dois 4-vetores momentum (também invariante de Lorentz) nos leva a relação de energiamomentum,

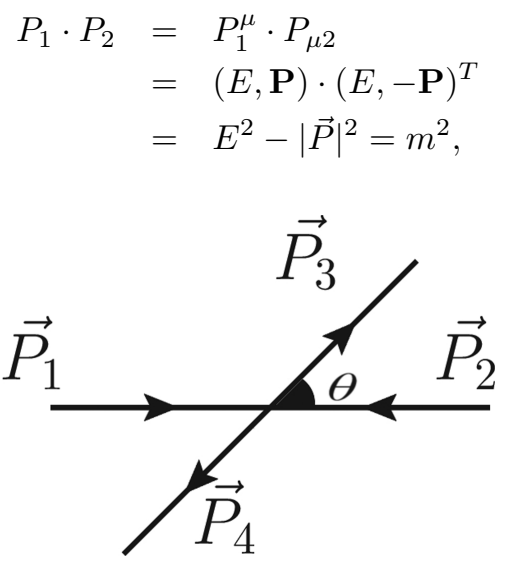

Figura 1: Representação do espalhamento $2 \rightarrow 2$ 
o que mostra que a massa é uma grandeza invariante. O último passo usa as definições para energia e momentum relativísticos. A partir destas relações entre os momenta iniciais, podemos construir relações úteis no referencial CM, também invariantes.

A forma como os 4-momenta são transferidos entre os estados inicial e final permitem definir grandezas invariantes, denominadas variáveis de Mandelstam. Elas apresentam relações que envolvem a energia, o momentum e o ângulo de espalhamento das partículas iniciais e finais do processo. Tomando uma das partículas (e seu momentum) como base, podemos ter três combinações, vistas como canais de transferência de momentum: aniquilação (canal s) e espalhamento (canais t e u), definidos como:

$$
\begin{aligned}
s & =\left(P_{1}+P_{2}\right)^{2}=\left(P_{3}+P_{4}\right)^{2} \\
t & =\left(P_{1}-P_{3}\right)^{2}=\left(P_{2}-P_{4}\right)^{2} \\
u & =\left(P_{1}-P_{4}\right)^{2}=\left(P_{2}-P_{3}\right)^{2}
\end{aligned}
$$

No limite relativístico, a massa invariante das partículas envolvidas no espalhamento Bhabha pode ser considerada como nula $\left(P_{i}^{2}=m_{i}^{2} \approx 0\right)$ e, aplicando a relação energia-momento 13 , obtemos

$$
\begin{aligned}
& s=P_{1}^{2}+P_{2}^{2}+2 P_{1} P_{2} \approx 2 P_{1} P_{2}=2\left(E^{2}-\overrightarrow{P_{1}} \cdot \overrightarrow{P_{2}}\right) \\
& s=2\left(E^{2}-|\vec{P}|^{2} \cos \theta\right)=4 E^{2}
\end{aligned}
$$

onde o ângulo entre os vetores momentum das partículas corresponde a 180 graus (pela conservação do momentum linear).

As demais variáveis de Mandelstam podem também ser definidas em termos do ângulo de espalhamento $\theta$, dadas por:

$$
\begin{aligned}
& s=4 E^{2} \\
& t=-2|\vec{P}|^{2}(1-\cos \theta)=-4 E^{2} \operatorname{sen}^{2}\left(\frac{\theta}{2}\right) \\
& u=-2|\vec{P}|^{2}(1+\cos \theta)=-4 E^{2} \cos ^{2}\left(\frac{\theta}{2}\right)
\end{aligned}
$$

Com isto, recuperamos, no limite relativístico $(m \approx 0)$, uma relação básica entre as variáveis de Mandelstam (garantida pela conservação do 4-momentum)

$$
s+t+u=4 m^{2} \text {. }
$$

Na próxima seção, vamos definir uma quantidade fundamental na Física de Partículas, uma vez que trabalhamos com espalhamentos e estes devem ter uma relação geométrica.

\section{Seção de choque}

A seção de choque é um parâmetro que informa a probabilidade de uma dada reação ocorrer, sendo este medido experimentalmente. Além disso, ela pode ser interpretada como uma área efetiva de interação e, portanto, medida em unidades de área. Pelas dimensões envolvidas na Física de Partículas, definimos uma unidade especial chamada barn, em que 1 barn $=10^{-28}$ metros $^{2}$.

Para determinar corretamente a seção de choque, devemos lembrar que a representação ocorre num espaço de transferência de momentum, chamado de espaço de fase do processo. Considerando um processo $1+2 \rightarrow 3+4$, existe uma taxa de transição por unidade de volume deste espaço de fase $W_{f i}$. Em outras palavras, esta é a densidade de probabilidade do processo ocorrer. A seção de choque é proporcional a esta densidade, considerando o fluxo de partículas incidentes e o número de estados detectados ao final do processo.

Considerando cada um destes termos em separado, iniciamos com o número de estados finais. Para definir o número total destes, vamos considerar uma porção finita do espaço de fase, uma caixa cúbica de lado $L$. Em cada dimensão linear, o número de estados possíveis é $\frac{L}{2 \pi} \Delta p$, onde o fator $2 \pi$ surge das condições de contorno periódicas do problema. Como este espaço considerado é isotrópico, o número total de estado disponíveis dentro desta caixa é $\frac{V}{(2 \pi)^{3}} \Delta^{3} p$. O número de partículas em cada unidade de volume é definido como $2 E$, de forma que o número de estados permitidos por partícula é

$$
\frac{V}{(2 \pi)^{3}} \frac{d^{3} p}{2 E} .
$$

O próximo passo é o cálculo do fluxo inicial de partìculas, onde podemos interpretar uma delas como projétil e outra como alvo. O fluxo relativo à partícula incidente, por unidade de volume é dado pelo produto entre sua velocidade e a densidade volumétrica; logo, $|\mathbf{v}| \frac{2 E}{V}$. Para o alvo, apenas nos interessa sua densidade $\frac{2 E}{V}$.

Para finalizar, precisamos determinar a densidade de probabilidade de ocorrência da colisão. Ela está associada à amplitude de espalhamento $\mathcal{M}$, em especial seu quadrado, tendo em vista que a densidade é um número real. Além disso, ela carrega um termo de conservação do 4-momentum, a função delta de Dirac, $\delta^{(4)}(p)$. Assim, pode ser expressa como

$$
W_{f i}=\frac{(2 \pi)^{4} \delta^{(4)}\left(p_{C}+p_{D}-p_{A}-p_{B}\right)}{V^{4}}|\mathcal{M}|^{2},
$$

onde temos a normalização por unidade de volume. Considerando o processo $A+B \rightarrow C+D$, podemos relacionar cada partícula a seu 4-momentum e temos

$$
\begin{aligned}
\Delta \sigma & =\frac{1}{\left|\mathbf{v}_{A}\right| 2 E_{A} 2 E_{B}} \frac{1}{(2 \pi)^{3}} \frac{d^{3} p_{C}}{2 E_{C}} \frac{1}{(2 \pi)^{3}} \frac{d^{3} p_{D}}{2 E_{D}}(2 \pi)^{4} \\
& \times \delta^{(4)}\left(p_{C}+p_{D}-p_{A}-p_{B}\right)|\mathcal{M}|^{2}
\end{aligned}
$$

onde definimos a seção de choque pela letra grega $\sigma$. Como o volume do espaço é arbitrário, ele é cancelado nesta expressão. Podemos identificar aqui três elementos, genericamente denotados por

$$
d \sigma=\frac{|\mathcal{M}|^{2}}{F} d \text { Lips }
$$


onde $F=\left|\mathbf{v}_{A}\right| 2 E_{A} 2 E_{B}$ e o elemento do espaço de fase invariante de Lorentz $d$ Lips $=(2 \pi)^{4} \delta^{(4)}\left(p_{C}+p_{D}-p_{A}-\right.$ $\left.p_{B}\right) \frac{d^{3} p_{C}}{(2 \pi)^{3} 2 E_{C}} \frac{d^{3} p_{D}}{(2 \pi)^{3} 2 E_{D}}$. Estes dois termos podem ser calculados e demonstram ser manifestamente invariantes. A informação física sobre o processo reside apenas na amplitude de espalhamento.

No referencial de centro de massa, onde os vetores momentum são simétricos, os fatores invariantes são simplificados em termos de ângulo de espalhamento e variáveis de Mandelstam

$$
\begin{aligned}
d \operatorname{Lips} & =\frac{1}{\left(4 \pi^{2}\right)} \frac{\left|\vec{p}_{f}\right|}{4 \sqrt{s}} d \Omega \\
F & =4\left|\vec{p}_{i}\right| \sqrt{s}
\end{aligned}
$$

onde $\left|\vec{p}_{i}\right|=\left|\vec{p}_{1,2}\right|$ e $\left|\vec{p}_{f}\right|=\left|\vec{p}_{3,4}\right|$.Assim, a seção de choque diferencial (dependente do ângulo de espalhamento $\theta$ ) para o processo é dada por [24]:

$$
d \sigma=\frac{\left|\vec{p}_{f}\right|}{\left|\vec{p}_{i}\right|} \frac{<|\mathcal{M}|^{2}>}{64 \pi^{2} s} d \Omega
$$

Como esta colisão é elástica (e a conservação de momentum linear nos garante que $\left.\left|\vec{p}_{f}\right|=\left|\vec{p}_{i}\right|\right)$, temos $E_{1}=E_{2}$. Logo, $s=\left(E_{1}+E_{2}\right)^{2}$ e:

$$
\frac{d \sigma}{d \Omega}=\frac{<|\mathcal{M}|^{2}>}{64 \pi^{2}(2 E)^{2}} .
$$

Para obtermos a predição dada pela teoria, resta-nos determinar o termo $|\mathcal{M}|^{2}$, associado à amplitude do espalhamento, calculado pela teoria e que nos informa a intensidade da interação.

Richard Phillips Feynman ${ }^{3}$ mostrou como calcular estas amplitudes a partir de representações gráficas, com regras definidas na Teoria Quântica de Campos para estas interações entre partículas, tratando estas representações não no espaço de posições, mas no espaço de momentum, ou seja, ilustrando graficamente o fluxo do momentum. Partindo dos diagramas de Feynman de um dado processo e usandos essas regras, apresentadas na Figura (2), conseguimos obter a amplitude invariante de Lorentz. Na próxima seção, utilizaremos estas regras para determinar a amplitude $\mathcal{M}$.

\section{O Processo Bhabha}

Conforme mencionado na introdução, o processo estudado por Bhabha 25] é o espalhamento elétron-pósitron $\left(e^{-}+e^{+} \rightarrow e^{-}+e^{+}\right)$. Para este processo temos a contribuição de dois diagramas, conforme podemos ver na Fig. (3). O diagrama 1, corresponde ao processo de aniquilação, e o diagrama 2 ao de espalhamento.

A amplitude total de Lorentz para este processo considera as contribuições dos dois diagramas, bem como de um termo de interferência entre eles. Este termo tem

$\sqrt[3]{\text { http://www-history.mcs.st-and.ac.uk/Biographies/Feynman. }}$ html

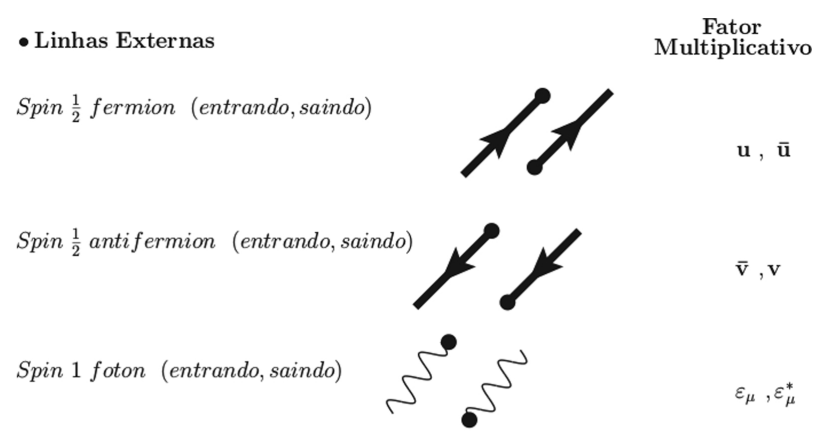

-Linhas Internas - Propagadores(sem a prescricao $+\mathrm{i} \varepsilon$ )

Spin $\frac{1}{2}$ fermion

Spin 1 boson foton (sem massa, Gauge de Feynman)

W

-Fatores de Vertice

Foton $-\operatorname{spin} \frac{1}{2}($ carga $-e)$

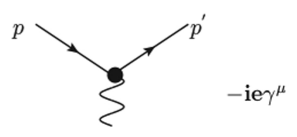

Figura 2: Regras de Feynman para QED 23.

contribuição negativa devido ao Princípio da Exclusão de Pauli.

Vamos utilizar as regras apresentadas na figura 2 a fim de obter a amplitude de Lorentz referente ao diagrama 1. Para analisar o gráfico devemos perceber que as linhas contínuas representam os férmions (elétrons ou pósitrons) e a seta indicativa do momentum diferencia ambos. $\mathrm{Ou}$ seja, considerando o tempo a partir da esquerda, $P_{1}$ e $P_{3}$ representam elétrons e $P_{2}$ e $P_{4}$ representam pósitrons. A leitura deste diagrama, usando as regras ilustradas na Fig. 2, nos fornece

$$
\mathcal{M}_{1}=\frac{-e^{2}}{s}\left[\bar{u}(3)\left(\gamma^{\mu}\right) v(4)\right]\left[\bar{v}(2)\left(\gamma_{\mu}\right) u(1)\right]
$$

e sua conjugada

$$
\mathcal{M}_{1}^{*}=\frac{-e^{2}}{s}\left[\bar{v}(4)\left(\gamma_{\mu}\right) u(3)\right]\left[\bar{u}(1)\left(\gamma^{\mu}\right) v(2)\right]
$$

A troca de momentum desse diagrama ocorre no canal s, ou seja, $s=\left(P_{1}+P_{2}\right)^{2}$.

A média quadrática da amplitude invariante de Lorentz é definida por:

$$
<|\mathcal{M}|^{2}>=\frac{1}{4}|\mathcal{M}|\left|\mathcal{M}^{\dagger}\right|
$$



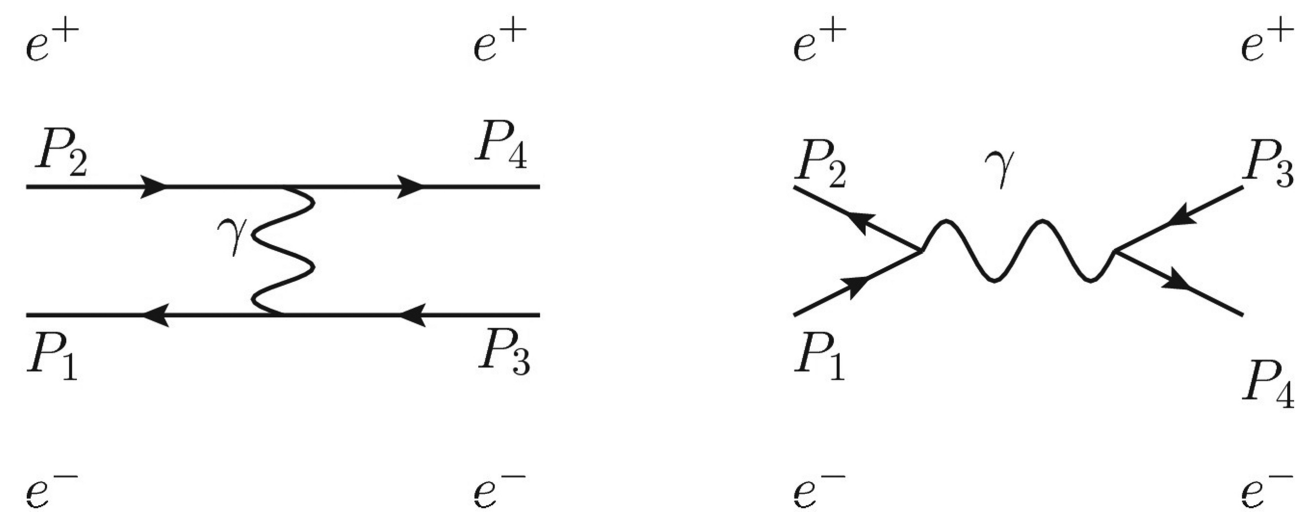

Figura 3: Canais com as contribuições de aniquilação (canal $s$ ) e espalhamento (canal $t$ ) para o processo Bhabha

onde o fator $\frac{1}{4}$ surge devido à média sobre todas as possíveis configurações de spins das partículas incidentes ${ }^{4}$

Com isso, e utilizando as relações de completeza presente no apêndice A1.1 e A1.2, tanto para o pósitron quanto para o elétron 5 , obtemos

$<\left|\mathcal{M}_{1}\right|^{2}>=\frac{8 e^{4}}{\left(P_{1}+P_{2}\right)^{4}}\left[\left(p_{1} \cdot p_{3}\right)\left(p_{2} \cdot p_{4}\right)+\left(p_{1} \cdot p_{4}\right)\left(p_{2} \cdot p_{3}\right)\right]$ (30)

na aproximação relativística que estamos utilizando. Reescrevendo esta expresão em termos das variáveis de Mandelstam (Eqs. 15), encontramos

$$
<\left|\mathcal{M}_{1}\right|^{2}>=2 e^{4}\left[\frac{t^{2}+u^{2}}{s^{2}}\right]
$$

No caso do diagrama 2 , onde a troca de momentum desse diagrama ocorre no canal t, ou seja, $t=\left(P_{1}-P_{3}\right)^{2}$, temos

$$
\mathcal{M}_{2}=\frac{-e^{2}}{k^{2}}\left[\bar{u}(3)\left(\gamma^{\nu}\right) u(1)\right]\left[\bar{v}(2)\left(\gamma_{\nu}\right) v(4)\right]
$$

e sua conjugada

$$
\mathcal{M}_{2}^{*}=\frac{-e^{2}}{t}\left[\bar{u}(1)\left(\gamma^{\nu}\right) u(3)\right]\left[\bar{v}(4)\left(\gamma_{\nu}\right) v(2)\right]
$$

Então, de forma similar ao cálculo que levou a Eq. 31 . obtemos

$$
<\left|\mathcal{M}_{2}\right|^{2}>=\frac{8 e^{4}}{\left(P_{1}-P_{3}\right)^{4}}\left[\left(p_{1} \cdot p_{4}\right)\left(p_{2} \cdot p_{3}\right)+\left(p_{1} \cdot p_{2}\right)\left(p_{3} \cdot p_{4}\right)\right]
$$

ou, em termos das variáveis de Mandelstam

$$
<\left|\mathcal{M}_{2}\right|^{2}>=2 e^{4}\left[\frac{u^{2}+s^{2}}{t^{2}}\right] .
$$

\footnotetext{
${ }^{4}$ A média é dada por $\frac{1}{\left(2 S_{1}+1\right)\left(2 S_{2}+1\right)}$ onde $S_{i}$ é o spin da partícula incidente i. Neste caso em particular, o spin do elétron é $1 / 2$. O spin do pósitron é idêntico ao do elétron. Logo, $S_{1}=S_{2}=1 / 2$, resultando no fator $1 / 4$.

${ }^{5}$ Os espinores $u$ e $v$ satisfazem a equação de Dirac no espaço de momento. Tendo as seguintes características: são ortogonais $\left(\bar{u}^{1} u^{2}=0\right.$ e $\left.\bar{v}^{1} v^{2}=0\right)$ e normalizados $\left(\bar{u}^{1} u^{1}=2 m c\right.$ e $\bar{v}^{1} v^{1}=$ $-2 m c)$.
}

Podemos verificar que os dois diagramas têm expressões similares finais, apenas mediante a troca $s \leftrightarrow t$. Esta observação é importante para que possamos verificar que os dois termos presentes na interferência terão este mesmo comportamento.

Para concluir o cálculo deste processo, devemos obter s termo de interferência. Na primeira parte dele, juntamos as expressões já definidas acima e temos

$$
\begin{aligned}
\mathcal{M}_{1} \mathcal{M}_{2}^{*} & =\frac{e^{4}}{t s} \underbrace{\left[\bar{u}(3)\left(\gamma^{\mu}\right) v(4)\right]}_{a} \underbrace{\left[\bar{v}(2)\left(\gamma_{\mu}\right) u(1)\right]}_{b} \\
& \times \underbrace{\left[\bar{u}(1)\left(\gamma^{\nu}\right) u(3)\right]}_{c} \underbrace{\left[\bar{v}(4)\left(\gamma_{\nu}\right) v(2)\right]}_{d}
\end{aligned}
$$

Como a,b,c e d são números, vamos reordená-los de maneira conveniente. Logo:

$$
\begin{aligned}
\mathcal{M}_{1} \mathcal{M}_{2}^{*} & =\frac{e^{4}}{t s}[a \cdot d \cdot b \cdot c]=\frac{e^{4}}{t s} \underbrace{\left[\bar{u}(3)\left(\gamma^{\mu}\right) v(4)\right]}_{a} \\
& \times \underbrace{\left[\bar{v}(4)\left(\gamma_{\nu}\right) v(2)\right]}_{d} \underbrace{\left[\bar{v}(2)\left(\gamma_{\mu}\right) u(1)\right]}_{b} \underbrace{\left[\bar{u}(1)\left(\gamma^{\nu}\right) u(3)\right]}_{c}
\end{aligned}
$$

Somando sobre todos os spins e sobre a média dos spins das partículas incidentes, temos:

$$
<\left|\mathcal{M}_{1} \mathcal{M}_{2}^{*}\right|>=\sum_{s} \sum_{s} \sum_{s} \sum_{s} \frac{1}{4}\left|\mathcal{M}_{1} \mathcal{M}_{2}^{*}\right|
$$

Como estamos trabalhando com números, conforme mostramos acima para rearranjá-los, esta expressão pode ser substituída usando a definição de traço de matriz (apêndice A.2.3) e a relação de completeza (apêndice A.1.1 e A.1.2)

$$
\begin{aligned}
<\left|\mathcal{M}_{1} \mathcal{M}_{2}^{*}\right|> & =\frac{1}{4} \frac{e^{4}}{t s} \operatorname{Tr}[\underbrace{u(3) \bar{u}(3)}_{D_{3}+m} \gamma^{\mu} \underbrace{v(4) \bar{v}(4)}_{D_{4}-m} \gamma_{\nu} \\
& \times \underbrace{v(2) \bar{v}(2)}_{D_{2}-m} \gamma_{\mu} \underbrace{u(1) \bar{u}(1)}_{D_{1}+m} \gamma^{\nu}]
\end{aligned}
$$

onde usamos a propriedade cíclica do traço para deslocar $u(3)$ para o início do termo. No limite relativístico, que 
utilizamos, $m \approx 0$ e temos:

$$
<\left|\mathcal{M}_{1} \mathcal{M}_{2}^{*}\right|>=\frac{1}{4} \frac{e^{4}}{t s} \operatorname{Tr}\left[\left(P_{3} \gamma^{\mu} P_{4} \gamma_{\nu} P_{2} \gamma_{\mu} P_{1} \gamma^{\nu}\right)\right]
$$

Aplicando novamente a propriedade do traço (apêndice A.2.3) na equação anterior, temos:

$$
\begin{aligned}
\left\langle\left|\mathcal{M}_{1} \mathcal{M}_{2}^{*}\right|>\right. & \left.=\frac{1}{4} \frac{e^{4}}{t s} \operatorname{Tr}[\underbrace{\left(D_{3} \gamma^{\mu} P_{4} \gamma_{\nu} P_{2}\right.}_{A} \underbrace{\gamma_{\mu} P_{1} \gamma^{\nu}}_{B})\right] \\
& =\frac{1}{4} \frac{e^{4}}{t s} \operatorname{Tr}[\underbrace{\gamma_{\mu} P_{1} \gamma^{\nu}}_{B} \underbrace{D_{3} \gamma^{\mu} P_{4} \gamma_{\nu} P_{2}}_{A}] \\
\left\langle\left|\mathcal{M}_{1} \mathcal{M}_{2}^{*}\right|>\right. & =\frac{1}{4} \frac{e^{4}}{t s} \operatorname{Tr}[\underbrace{\gamma_{\mu} P_{1} \gamma^{\nu} P_{3} \gamma^{\mu}}_{C} P_{4} \gamma_{\nu} P_{2}]
\end{aligned}
$$

Aplicando a propriedade da notação slash(apêndice A.3.8') em C da Eq 36 .

$$
<\left|\mathcal{M}_{1} \mathcal{M}_{2}^{*}\right|>=\frac{1}{4} \frac{e^{4}}{t s}(-2) \operatorname{Tr}[P_{3} \underbrace{\gamma^{\nu} P_{1} P_{4} \gamma_{\nu}}_{D} P_{2}]
$$

Fazendo uso da propriedade das matrizes gama(apêndice A.3.7) em $\mathrm{D}^{6}$ na equação acima:

$$
<\left|\mathcal{M}_{1} \mathcal{M}_{2}^{*}\right|>=\frac{1}{4} \frac{e^{4}}{t s}\left(-8\left(P_{1} \cdot P_{4}\right) \operatorname{Tr}\left[P_{3} P_{2}\right]\right.
$$

Por fim, aplicando o teorema do traço (apêndice A.4.4), obtemos

$$
<\left|\mathcal{M}_{1} \mathcal{M}_{2}^{*}\right|>=\frac{1}{4} \frac{e^{4}}{t s}(-32)\left(P_{1} \cdot P_{4} \cdot P_{3} \cdot P_{2}\right)
$$

ou, em termos das variáveis de Mandelstam

$$
-<\left|\mathcal{M}_{1} \mathcal{M}_{2}^{*}\right|>=2 e^{4} \frac{u^{2}}{t s}
$$

Como a troca do diagrama 1 pelo 2 levou a resultados similares em suas amplitudes de espalhamento, exceto pela troca $s \leftrightarrow t$, podemos considerar a mesma troca para a segunda parte do termo de interferência, o que leva a um valor igual ao obtido no primeiro. Assim, a soma deles resulta em:

$$
-\left(<\left|\mathcal{M}_{1} \mathcal{M}_{2}^{*}\right|>+<\left|M_{1}^{*} M_{2}\right|>\right)=4 e^{4} \frac{u^{2}}{t s}
$$

Com isso, a amplitude total de Lorentz é:

$$
<|\mathcal{M}|^{2}>=2 e^{4}\left[\frac{t^{2}+u^{2}}{s^{2}}+\frac{u^{2}+s^{2}}{t^{2}}+2 \frac{u^{2}}{t s}\right]
$$

Substituindo as variáveis de Mandelstam (Eq. 15 por sua relação com os ângulos de espalhamento, a Eq. (39) torna-se

$$
\begin{aligned}
<|\mathcal{M}|^{2}> & =2 e^{4}\left[\frac{\cos ^{4}\left(\frac{\theta}{2}\right)+1}{\operatorname{sen}^{4}\left(\frac{\theta}{2}\right)}+\frac{\left(1+\cos ^{2}(\theta)\right)}{2}\right. \\
& \left.-2 \frac{\cos ^{4}\left(\frac{\theta}{2}\right)}{\operatorname{sen}^{2}\left(\frac{\theta}{2}\right)}\right]
\end{aligned}
$$

\footnotetext{
${ }_{6}$ Lembrando que agora $P_{1} P_{2}$ são escalares e, portanto, saem do traço.
}

Usando as relações trigonométricas e a substituição $x=\cos \theta$, podemos reescrever como

$$
<|\mathcal{M}|^{2}>=2 e^{4}\left[\frac{(1+x)^{2}+4}{(1-x)^{2}}+\frac{\left(1+x^{2}\right)}{2}-\frac{(1+x)^{2}}{(1-x)}\right]
$$

Aplicando a expressão acima na Eq. 26, obtemos

$$
\frac{d \sigma}{d \Omega}=\frac{2 e^{4}}{64 \pi^{2}(2 E)^{2}}\left[\frac{(1+x)^{2}+4}{(1-x)^{2}}+\frac{\left(1+x^{2}\right)}{2}-\frac{(1+x)^{2}}{(1-x)}\right]
$$

usando a definição da constante de estrutura fina $(\alpha=$ $\left.\frac{e^{2}}{4 \pi} \approx \frac{1}{137}\right)$ e de energia de centro de massa $(\sqrt{s}=$ $\left.2 E_{\text {feixe }}\right)$ :

$$
\frac{d \sigma}{d \Omega}=\frac{\alpha^{2}}{2 s}\left[\frac{(1+x)^{2}+4}{(1-x)^{2}}+\frac{\left(1+x^{2}\right)}{2}-\frac{(1+x)^{2}}{(1-x)}\right]
$$

A figura 5 mostra a seção de choque diferencial calculada através da Eq. 40 Nesta figura foi comparada a curva teórica com os dados obtidos pela Colaboração Tassd 7 . Ainda, se a energia de centro de massa fosse da ordem da massa do bóson $\mathrm{Z}\left(m_{Z}=91.1876 \mathrm{GeV}\right)[24]$, teríamos um diagrama idêntico ao do canal s exceto pela troca de $\gamma$ por Z. O bóson $\mathrm{Z}$ (neutro) e $W^{ \pm}$(carregado) são bósons massivos descritos pela teoria Eletrofraca. Sendo esta responsável pelo decaimentos radioativos. Desta forma, teríamos 3 diagramas mais as suas respectivas interferências.

Para obtermos a figura 4, os dados experimentais devem ser dividos por s (energia de centro de massa ao quadrado), pois é desta forma que os dados experimentais estão no artigo experimental.

Além disso, integrando a equação(35) no ângulo sólido $|\cos (\theta)|<0.84$, obtemos a seção de choque total em relação a energia de centro de massa, como é mostrado na figura 5.

Este resultado, confrontando teoria e experimento, é a principal avaliação de nosso cálculo. Antes de afirmarmos a boa descrição dos dados experimentais, é importante ressaltar que esta etapa nos permite grandes avanços na teoria e na fenomenologia da Física de Partículas. Esta comparação deve ser vista, então, como o principal resultado de qualquer cálculo de processos na Física de Altas Energias.

\subsection{Simetria de Cruzamento}

A simetria de cruzamente ${ }^{8}$ pode ser usada para se obter a amplitude de espalhamento através de outro processo, ou seja, trocando a partícula $\mathrm{P}$ pela sua anti-partícula $\bar{P}$. Além disso, também permite a obtenção de um decaimento do processo $A \rightarrow \bar{B}+C+D$ a partir de $A+B \rightarrow$ $C+D$. Cabe salientar que o número leptônico sempre

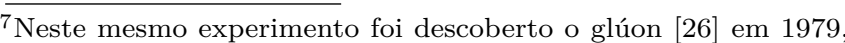
o mediador da força forte, descrito pela cromodinâmica quântica(Quantum Cromodynamics - QCD).

${ }^{8} \mathrm{Ou}$ crossing symmetry.
} 


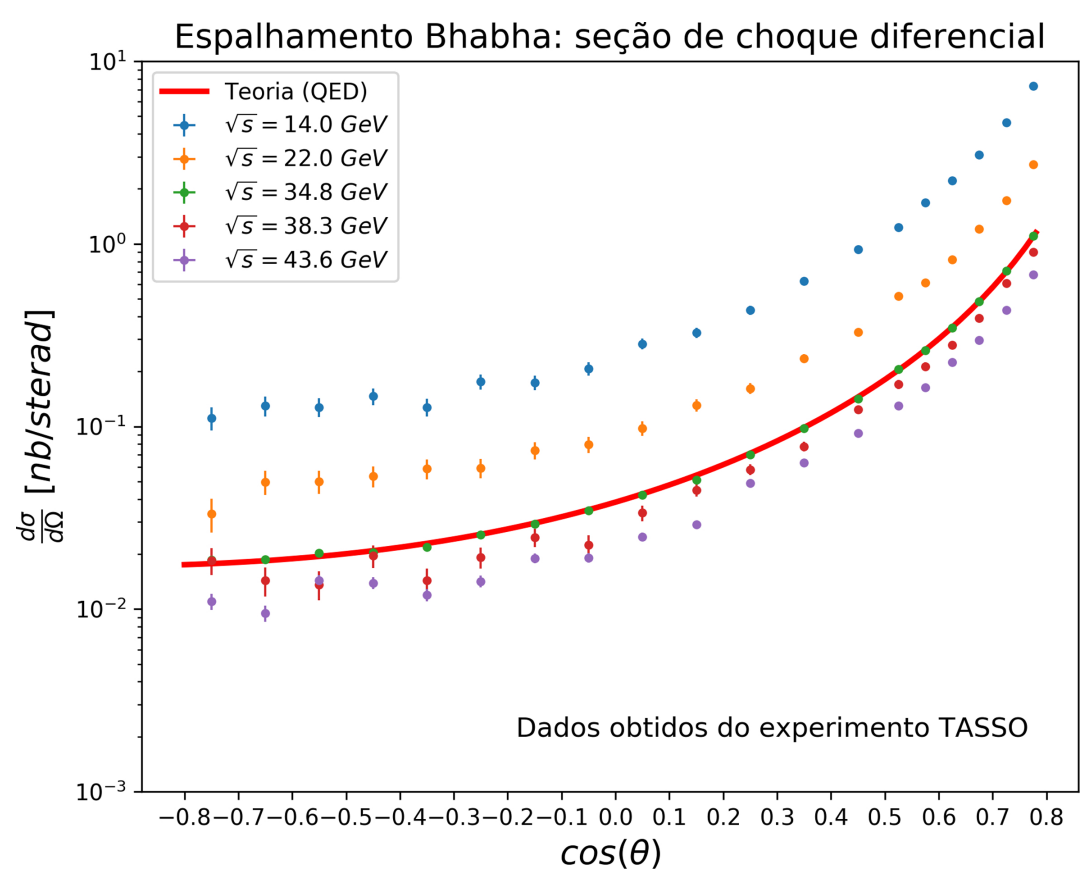

Figura 4: Seção de choque diferencial em termos do $\cos \theta$.

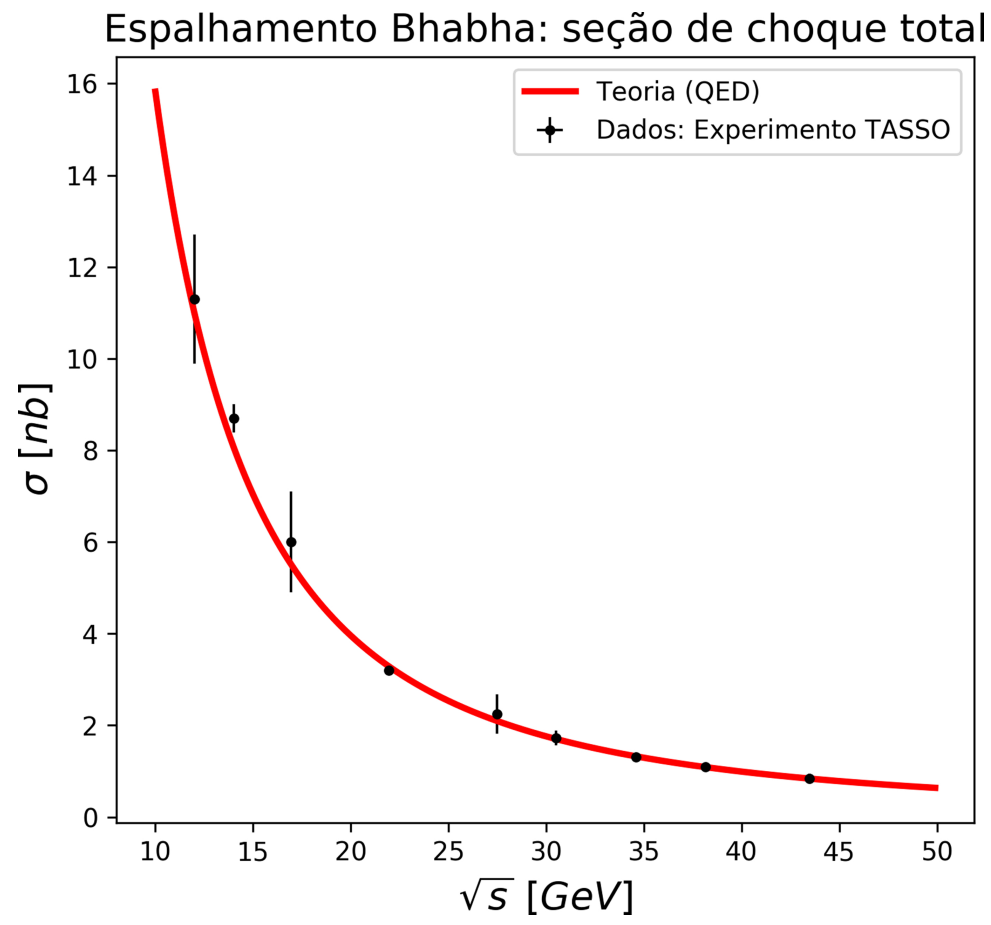

Figura 5: Seção de choque total em termos da energia de centro de massa $(\sqrt{s})$.

deve ser conservado. Um exemplo prático da simetria de crossing é a obtenção da amplitude de $e^{-} \mu^{-} \rightarrow e^{-} \mu^{-}$a partir da amplitude do Bhabha no canal S. Neste processo temos $e^{-}\left(P_{1}\right)+e^{+}\left(P_{2}\right) \rightarrow e^{+}\left(P_{3}\right)+e^{-}\left(P_{4}\right)$. Por não termos aniquilação de partículas e por serem de sabores diferentes, teremos somente a contribuição no canal t. Para tanto, substituindo $e^{+}\left(P_{2}\right) \rightarrow \mu^{-}\left(-P_{3}\right)$ na eq. 31 . obteremos a amplitude da eq. 35 a qual corresponde a amplitude do processo $e^{-} \mu^{-} \rightarrow e^{-} \mu^{-}$.

\section{Considerações Experimentais}

Para checarmos a qualidade da teoria contra experimento, utilizamos o teste do qui-quadrado $\left(\chi^{2}\right)$ e chi-quadrado reduzido $\left(\chi^{2} / \nu\right)$. As definições destes podem ser vistas no apêndice A.5. Para realizar esse teste, usamos a energia de centro de massa $(\sqrt{s}=34.8 \mathrm{GeV})$, com luminosidade integrada $\left(\int \mathcal{L} d t=174.5 \mathrm{pb}^{-1}\right)$ correspondendo a 166348 eventos, sendo este o maior número de eventos do ar- 
tigo 27. Obtemos $\chi^{2}=23.89$ com número de graus de liberdade $(\mathrm{ndf}=\nu)$ igual a 19 . Para o $\chi^{2} / \nu=1.25$. Um bom ajuste é aquele em que o $\left(\chi^{2} / \nu\right)$ aproximasse de 1 . A probabilidade de obtermos o mesmo resultado ou uma resultado maior numa amostra qualquer é de $\mathrm{P}\left(\chi^{2}, \nu\right)=20 \%$. Onde o ideal é quando $\mathrm{P}\left(\chi^{2}, \nu\right)$ aproximasse de $50 \%$ [28].

\section{Considerações Finais}

Neste trabalho, buscamos apresentar algumas das técnicas utilizadas na Física de Partículas de forma didática, visando fornecer aos estudantes dos últimos anos dos cursos superiores uma visão da física e da matemática envolvidas nos tópicos mais avançados. Com a entrada em funcionamento do LHC, em 2010, e com o anúncio da descoberta do Bóson de Higgs, em 4 de Julho 2012, muitos acadêmicos trazem suas curiosidade ao ingressarem na graduação. Aqui, mostramos através do cálculo de um processo específico, como os físicos tratam as colisões num experimento, e a partir desse tratamento, fazem as predições sobre resultados para cada processo.

A escolha do processo Bhabha deu-se, em primeiro lugar, por trabalhar com muitos dos aspectos fundamentais do cálculo de processos, porém numa interação limpa, que é a eletromagnética, descrita pela Eletrodinâmica Quântica. Além de seu caráter didático para o cálculo de amplitudes, o processo Bhabha é frequentemente usado como um monitoramento da luminosidade [1] para experimentos envolvendo espalhamentos elétron-pósitron. Medidas precisas da luminosidade são necessárias para diminuir os erros experimentais na medição das seções de choque.

Finalmente, entendemos que apresentar tópicos mais avançados da Física, cujo caráter se aproxima mais da pesquisa do que do ensino, em um nível suficientemente claro para ser lido e estudado por acadêmicos nos últimos anos, é uma estratégia para despertar o talento e a curiosidade precoces nestes, fomentando as oportunidades de formação nesta área. Os scripts (Mathematica, Gnuplot e Python) e o código em FORTRAN estão disponíveis online 9

\section{Agradecimentos}

F. K. N. e L. F. M. agradecem ao revisor deste artigo pelas apropriadas recomendações. Por fim, F.K.N. agradece ao CNPq pelo suporte financeiro.

\section{Material Suplementar}

O seguinte material suplementar está disponível online: Apêndice A

\section{Referências}

[1] I. Bozovic-Jelisavcic, S. Lukic, G. Milutinovic Dumbelovic, M. Pandurovic e I. Smiljanic, Journal of Instrumentation 8, P08012 (2013).

[2] A.C.B. Machado, V. Pleitez e M.C. Tijero, Rev. Bras. Ens. Fís. 28, 407 (2006).

[3] G. Brockington e M. Pietrocola, Inv. Ens. Cien. 10, 387 (2005).

[4] S. Tomonaga, Prog. Theo. Phys. 1, 27 (1948).

[5] J. Schwinger, Phys. Rev. 73, 416 (1948).

[6] J. Schwinger, Phys. Rev. 74, 1439 (1948).

[7] R. P. Feynman, Phys. Rev. 76, 749 (1949).

[8] R. P. Feynman, Phys. Rev. 76, 769 (1949).

[9] R. P. Feynman, Phys. Rev. 80, 440 (1950)

[10] W. B. de Sousa, Física das Radiações: uma proposta para o Ensino Médio. Dissertação de Mestrado, Universidade de São Paulo, 2009.

[11] J. M. F. Bassalo, Eletrodinâmica Quântica (Livraria da Física, São Paulo, 2006).

[12] B. R. Martin e G. Shaw, Particle Physics (Wiley, Chichester, 2008), 4 ed.

[13] M. Thompson, Modern Particle Physics (Cambridge University Press, Cambridge, 2013), 1 ed.

[14] A. Bettini, Introduction to Elementary Particle Physics (Cambridge University Press, Cambridge, 2012), 2 ed.

[15] D. Griffths, Introduction to Elementary Particles (Wiley, Weinheim, 2008), 2 ed.

[16] H. V. Fagundes, Teoria da Relatividade no Nível Matemático do Ensino Médio (Editora Livraria da Física, São Paulo, 2009).

[17] R. D'Auria e M. Trigiante, From Special Relativity to Feynman Diagrams: A Course in Theoretical Particle Physics for Beginners (Springer, Switzerland, 2016), 2 ed.

[18] J. H. Smith, Introduction to Special Relativity (Dover Publications, Dover, 2015), 1 ed.

[19] Y. Nagashima, Elementary Particle Physics: Quantum Field Theory and Particles (Wiley, Weinheim, 2010), v. 1, p. 964.

[20] M. E. Peskin e D. V. Schroeder, An Introduction to Quantum Field Theory (Perseus, Reading, 1995), 1 ed.

[21] M. D. Schwartz, Quantum Field Theory and the Standard Model (Cambridge University Press, Cambridge, 2014), 1 ed.

[22] T. Lancaster e S. J. Blundell, Quantum Field Theory for the Gifted Amateur (Oxford University Press, Oxford, 2014).

[23] F. Halzen e A. D. Martin, Quarks and Leptons: An Introductory Course in Modern Particle Physics (Wiley, New York, 1984), 1 ed.

[24] C. Patrignani et al. (Particle Data Group), Chin. Phys. C 40, 100001 (2016).

[25] H. J. Bhabha, Proc. Roy. Soc. A154, 195 (1935).

[26] J. Ellis, Int. J. Mod. Phys. A29, 1430072 (2014).

[27] W. Braunschweig et al. (TASSO Coll.), Z. Phys. C37, 171 (1988).

[28] P. R. Bevington e D. K. Robinson, Data Reduction and Error Analysis for the Physical Sciences (McGraw-Hill, New York, 2002), 3 ed. 